\title{
Multilevel analysis of the role of women's empowerment on use of contraceptive methods among married Cambodian women: evidence from demographic health surveys between 2005 and 2014
}

\author{
Owen Nkoka ${ }^{1,2}$, Daphne Lee ${ }^{1}$, Kun-Yang Chuang ${ }^{1}$ and Ying-Chih Chuang ${ }^{1 *}$ (D)
}

\begin{abstract}
Background: The use of contraceptives is an essential public health concept that improves overall safe motherhood and infant health. Women empowerment has been reported to influence health behaviors in women. With recent efforts to increase access to contraceptive methods, uptake of the same remains a challenge in Cambodia. There are limited studies that have examined the role of women's empowerment at both individual- and community- level on contraceptive use in Cambodia. This study examined the individual- and community-level factors associated with contraceptive use among Cambodian married women between 2005 and 2014.
\end{abstract}

Methods: Data from 2005, 2010, and 2014 Cambodia Demographic and Health Surveys were used to analyze 2211; 10,505; and 10,849 women, respectively. Multilevel binary and multinomial logistic regression models were applied to assess the association between individual- and community- level factors, and the use of contraceptive methods.

Results: The prevalence of using modern contraceptive methods increased over time (i.e., 29.0, 38.1, and $42.3 \%$ in 2005, 2010, and 2014, respectively). At the individual level, women who attained secondary and higher education were more likely to use any contraceptives [adjusted odds ratio $(\mathrm{aOR})=1.43,95 \%$ confidence interval $(\mathrm{Cl})=1.22-1.68$, and $\mathrm{aOR}=1.23,95 \% \mathrm{Cl}=1.05-1.44$ in 2010 and 2014, respectively] compared with those with no formal education. Similarly, having a high workforce participation level was significantly associated with increased likelihood of using any contraceptive methods $[\mathrm{aOR}=1.12,95 \% \mathrm{Cl}=1.00-1.26, \mathrm{aOR}=1.44,95 \% \mathrm{Cl}=1.29-1.60$ and in 2010 and 2014, respectively]. Other factors such as age at first marriage, residence, and having a health insurance were associated with contraceptive use. The proportional change in variance showed that about $14.3 \%$ of total variations in the odds of contraceptive use across the communities were explained by both individual- and community-level factors. Moreover, the intraclass correlation showed that about $5.2 \%$ of the total variation remained unexplained even after adjustments.

Conclusion: Both individual- and community- level factors influenced contraceptive use in Cambodia. When designing programs to improve contraceptive use, contextual influences should be taken into account for the effectiveness of the programs.

\footnotetext{
*Correspondence: yingchih@tmu.edu.tw

1 School of Public Health, Taipei Medical University, Taipei City, Taiwan 110

Full list of author information is available at the end of the article
} original author(s) and the source, provide a link to the Creative Commons licence, and indicate if changes were made. The images or other third party material in this article are included in the article's Creative Commons licence, unless indicated otherwise in a credit line to the material. If material is not included in the article's Creative Commons licence and your intended use is not permitted by statutory regulation or exceeds the permitted use, you will need to obtain permission directly from the copyright holder. To view a copy of this licence, visit http://creativecommons.org/licenses/by/4.0/. The Creative Commons Public Domain Dedication waiver (http://creativeco mmons.org/publicdomain/zero/1.0/) applies to the data made available in this article, unless otherwise stated in a credit line to the data. 
Keywords: Women's empowerment, Contraceptive use, Multilevel analysis, Cambodia, DHS

\section{Background}

Contraceptive use is an important public health issue that promotes safe motherhood and infant health [1-3]. Contraceptives may be used for appropriate child spacing which has been shown to reduce the likelihood of preterm births $[4,5]$. Additionally, contraceptives may be used for limiting child bearing especially to avoid high risk pregnancies thereby, helping to reduce maternal and infant mortality [6, 7]. A multi-country study conducted in 2010 revealed that contraceptive use reduced maternal mortality globally by $44.0 \%$ [6]. Since 1987 , a global initiative known as "Safe Motherhood" has been championed to reduce maternal mortality [8-10]. This initiative encompasses a wide range of issues relating to maternal health including family planning (FP) - one of the four pillars of safe motherhood [11].

An important component of FP is the use of contraceptives. FP is not only a significant health issue but also affects a wide range of determinants essential for the attainment of sustainable development goals [12]. Therefore, more emphasis has been placed in making modern FP methods accessible to the population to ensure good FP practices $[13,14]$. However, the use of contraceptives has been shown to be influenced by a wide range of social, cultural, and religious factors [15-17]. One dimension that has been shown to influence contraceptive use is women empowerment. For instance, a study in lower middle income countries reported an association between a woman's decision making power within a household and the use of contraceptives [18]. Women with household decision-making power are more likely to have control of their bodies and fertility [19]. Further, attainment of high educational levels bequeaths women with knowledge and skills that promote their well-being [20]. Moreover, women's involvement in workforce improves their economic independence which may ultimately help them have better access to healthcare services including contraceptives. Thus, education, workforce and decision making power have been widely promoted as important measurements for women empowerment [21, 22].

Cambodia experiences high rates of fertility and maternal mortality [23]. Despite several FP programs aimed to make contraceptives accessible, uptake of contraceptives has been a challenge [24]. A study was conducted in Cambodia to understand influence of social networks and contraceptive use [25]. Additionally, a recent
Cambodian study revealed that women empowerment within the household was critical for the use of contraceptives [26]. To date, there is no study in Cambodia that examined the role of individual- and community- level factors in influencing contraceptive use. Further, few studies have examined empowerment at both individualand community- level and how this influences contraceptive use $[11,27]$. Community factors have been shown to influence health behaviors and access to services $[28,29]$. It is, therefore, important to understand the influences of community characteristics on contraceptive use as findings from this research may help design effective FP programs that take into account the contextual factors.

Therefore, this study examined the influence of individual- and community- level factors, with an emphasis on women's empowerment measures, on contraceptive use among married Cambodian women between 2005 and 2014.

\section{Methods}

\section{Study design and data source}

This cross-sectional study used data from the 2005, 2010, and 2014 Cambodia demographic and health surveys (CDHS). The surveys adopted a stratified twostage cluster sampling method to select households for the survey. The first stage involved the selection of enumeration areas (EAs) and household listing within the selected EAs. In the second stage, households were selected through equal probability sampling criterion. In 2005, 557 EAs were selected while 677 EAs were selected for both 2010 and 2014 surveys. Details of the sampling strategy of the 2005, 2010, AND 2014 CDHS have been published elsewhere [30-32]. A community was defined as the primary sampling unit (i.e., enumeration areas) of the CDHS. Face to face interviews were conducted to all women of the reproductive age (15-49years) in the sampled households. Data analyzed in this study were from the individual (women's) questionnaire of the CDHS which have been published elsewhere [20-32]. In 2005, of the 17,256 eligible women, 16,823 were successfully interviewed representing a $98 \%$ response rate. The subsequent surveys also yielded a $98 \%$ response rate (i.e., 18,754 of 19,237 in 2010 and 17,578 of 18,012 in 2014). The current analysis was restricted to women who were currently married/in union or living with a man (i.e., $n=2211$ in $2005, n=10,505$ in 2010 , and $\mathrm{n}=10,849$ in 2014). 


\section{Measures \\ Outcome}

The dependent variable in this study was contraceptive use defined as the use of either traditional or modern contraceptive methods. Modern methods of contraception included pills, female and male sterilization, intrauterine device, injectable, implants, male and female condom, and the diaphragm [33-35]. On the other hand, traditional methods of contraception included withdrawal, periodic abstinence, and folk methods [3335]. Contraceptive use was categorized as a two-level (Any method and no method), and three-level (modern method, traditional method, and no method) variables.

\section{Independent variables - individual-level factors}

Three variables at individual-level were considered to measure women's empowerment status. First, educational level was categorized as "no formal", "primary", and "secondary and tertiary”. Second, women's household decision-making power was measured based on responses to individual questions regarding who has the final say in the family on the respondent's health care, large household purchases, and visits to family or relatives. Response options included (a) respondent alone, (b) respondent and husband/partner, (c) respondent and other person, (d) husband/partner alone, (e) someone else, and (f) other. For each question, a value of 1 was assigned for responses of (a), (b), or (c) to designate high decision-making power, and 0 for $(\mathrm{d}),(\mathrm{e})$, or $(\mathrm{f})$ to designate low power. A composite score was created for responses to the three dimensions of decision making power (i.e., health care, large household purchases, and visits to family/relatives) that ranged from 0 to 3. Participants were categorized as having low (a score of 0 to 1 ), middle (score of 2), and high (score of 3) household decision making power. Third, workforce participation consisted of current occupational status (yes or no), work consistency (respondent working throughout the year was coded as "1", those working seasonally or just occasionally were coded as "0"), and payment type (respondent who reported to receive cash was coded as " 1 " while those paid in-kind ora combination of the two or not paid for their work were coded as "0"). A score was generated and grouped into three levels; low "0 to 1 ", middle "score of 2 ", and high "score of 3 ".

Additionally, following a literature review [18, 36, 37], a wide range of individual-level factors were such as age (15-24, 25-34, or 35-49years), religion (Buddhist, Muslim, or other), place of residence (urban or rural), region (plains, Tonle Sap, plateau / mountain, or Phnom Penh), age at first marriage $(\leq 16,17-20$, or $\geq 21$ years $)$, total children ever born $(0,1-2$, or $\geq 3)$, distance to health facility (big problem or not), health insurance coverage (no or yes), and husband/partner educational attainment (no education, primary, or secondary and higher). The CDHS assessed wealth index as a composite score measured from household assets such as televisions, bicycles [30-32]. The scores were grouped into quintiles from poorest to richest. For purposes of this research, wealth was grouped into 3-levels as poor (lower 40\%), middle (middle 20\%), and rich (upper 40\%). Media exposure was measured by access to newspapers, radio, and television. Individuals reporting to have read newspaper, or watched television, or listened to radio at least once a week were categorized as having media exposure, otherwise, no.

\section{Independent variables - community-level factors}

The main independent variables at individual level were aggregated to form variables at community level. Community education, community workforce participation, community women's decision making power, and community wealth were calculated as proportions of women with any education, with workforce participation, having power to make decisions, and from rich households, respectively. The continuous variables were then grouped into tertiles as low, middle, and high for easy interpretation of the results.

\section{Data analysis}

Weighted frequencies and percentages were reported for the selected factors. The statistical software Stata was used for analysis of the datasets. The "svy" command was used to account for the sampling weights and clustering effects of the Demographic and Health Survey (DHS). The weights were calculated according to DHS guidelines [38]. Multilevel binary and multinomial logistic regression models were used to examine the association of individual- and community-level factors and contraceptive use. The binary multilevel logistic model was assessed using the "xtmelogit" command in Stata. The multinomial logistic model was estimated using generalized structural equation modeling (GSEM) using the "gsem" command. Four models were fitted in each survey year. Model 1 included outcome variable only, model 2 included the outcome and individual-level variables, model 3 included the outcome and community-level variables, and model 4 included the outcome and both individual and community-level factors. Fixed effects were reported as adjusted odds ratio (aOR) with 95\% confidence intervals (CIs). Random effects were reported as area variance, intraclass correlation coefficient (ICC), proportional change in variance $(\mathrm{PCV})$, and median odds ratio (MOR). Model goodness of fit was checked by Akaike information criterion (AIC) with lower AIC suggesting a better fit. All analyses were performed using Stata version 15 (Stata Corp, College Station, TX, USA) and significance level was set at $p<0.05$. 


\section{Ethical considerations}

The survey protocol was reviewed and approved by the Cambodia National Ethical Committee for Health Research. Informed consent for the surveys was obtained from each respondent at the start of each interview. Clearance to analyze the data was provided by the Demographic Health Survey (DHS) program. The data is publicly available and may be requested from the DHS program through https://dhsprogram .com/data/available-datasets.cfm.

\section{Results}

\section{Descriptive results}

The prevalence of contraceptive use is displayed in Fig. 1. Approximately $29.0 \%$ women reported using modern contraceptive methods in 2005 while $38.1 \%$ in 2010 and $42.3 \%$ in 2014 used modern contraceptive methods.

Table 1 lists the descriptive characteristics of 2211 women in 2005 (nested in 556 communities), 10,505 women in 2010 (nested in 611 communities), and 10,849 women in 2014 (nested in 611 communities). Among others, in all the three surveys, a majority of the women had primary education, high decision making power within their households, aged $\geq 35$ years, Buddhist, from rural areas, and had media exposure. Workforce participation increased over time with 25.0,
29.6, and $39.0 \%$ of the women having high workforce participation in 2005, 2010, and 2014, respectively.

\section{Empowerment factors associated with use of any contraceptive method}

Table 2 displays the adjusted effects of a wide range of individual- and community level factors on contraceptive use (i.e., any method vs no method). Results from model 4 have been emphasized as the model displayed better fit.

While educational level, women's decision making power, and workforce participation were not associated with use of any contraceptive method in 2005, significant associations were observed in 2010, and 2014. Specifically, in 2010, women with primary [aOR: 1.14, 95\% CI: 1.01-1.29] and secondary and tertiary [aOR: 1.43, 95\% CI: $1.22-1.68$ ] were more likely to use any contraceptive method compared with those having no formal education. Similarly, in 2014, compared with women with no formal education, increased odds were observed among those with primary [aOR: 1.17, 95\% CI: 1.02-1.34] and secondary and tertiary [aOR: $1.23,95 \% \mathrm{CI}$ : 1.05-1.44].

The results in 2010 further revealed that women with middle workforce [aOR: 1.14, 95\% CI: 1.01-1.28] and high [aOR: 1.12, 95\% CI: 1.00-1.26] participation levels were more likely to use any contraceptive methods compared with those having low workforce participation. In 2014, similar associations were noted with increased odds observed among women with middle [aOR: 1.27,

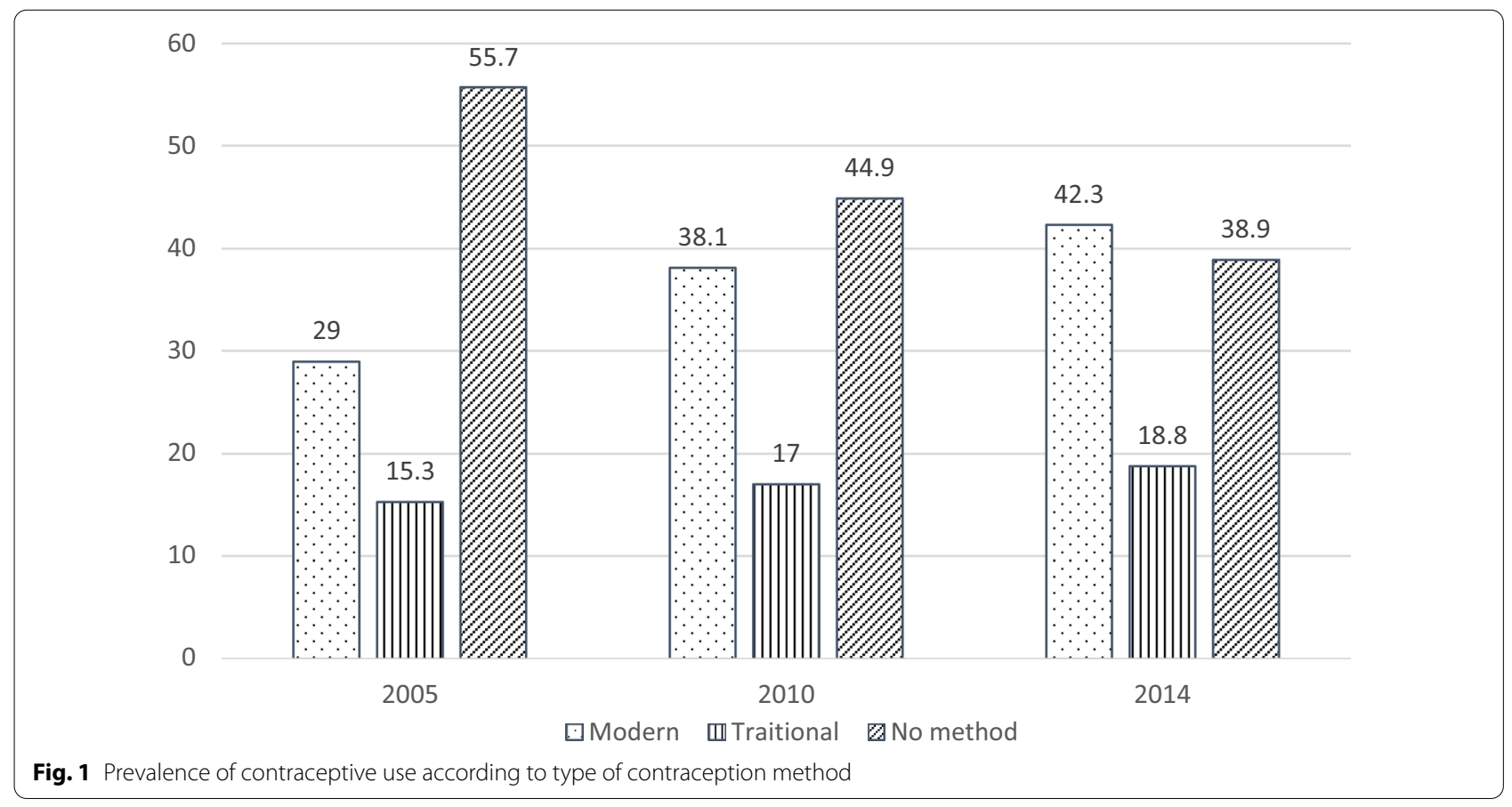


Table 1 Descriptive of individual- and community level characteristics of study sample

\begin{tabular}{llll}
\hline Variables & 2005 & 2010 & 2014 \\
& $(n=2211)$ & $(n=10,505)$ & $(n=10,849)$ \\
& $n(\%)$ & $n(\%)$ & $n(\%)$ \\
\hline
\end{tabular}

Educational level

No formal education

Primary

Secondary+

Decision-making

Low

High

Workforce participation

Low

Medium

High

Age (years)

$15-24$

25-34

$\geq 35$

Religion

Buddhist

Muslim

Other

Residence

Urban

Rural

Region

Plains

Tonle Sap

Plateau / Mountain

Phnom Penh

Age at first marriage

$\leq 16$
$17-20$
$\geq 21$

Total children ever born

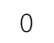

$1-2$

$\geq 3$

Wealth

Poor

Middle

Rich

Media exposure

No

Yes

Perceived distance to HF

Big problem

No problem

$\begin{array}{rrr}498(22.5) & 1995(19.0) & 1662(15.3) \\ 1323(59.8) & 5907(56.2) & 5886(54.3) \\ 390(17.7) & 2603(24.8) & 3301(30.4)\end{array}$

$92(4.2) \quad 400(3.8) \quad 382(3.5)$

$409(18.5) \quad 1056(10.1) \quad 1052(9.7)$

$1710(77.3) \quad 9049(86.1) \quad 9415(86.8)$

$890(40.2) \quad 4424(42.1) \quad 3393(31.3)$

$770(34.8)$

$551(25.0)$

368 (16.6)

$732(33.1)$

$1111(50.2)$

$2142(96.9)$

$39(1.8)$

$30(1.3)$

$353(16.0)$

$1858(84.0)$

$898(40.6)$

$834(37.7)$

$268(12.1)$

$211(9.6)$

$423(19.2)$

$1078(48.7)$

$710(32.1)$

$2972(28.3)$

$3109(29.6)$

$1615(15.4)$

$3898(37.1)$

$4992(47.5)$

$10,218(97.3)$

$146(1.4)$

$141(1.3)$

$1893(18.0)$

$8613(82.0)$

4345 (41.4)

$3695(35.2)$

$1448(13.8)$

$1017(6.6)$

$1928(18.3)$

$5070(48.3)$

3507 (33.4)

$$
82(3.7)
$$

788 (35.6)

$1341(60.7)$

444 (4.2)

$4450(42.4)$

$5611(53.4)$

$886(40.0)$

$4136(39.4)$

2059 (19.6)

$4310(41.0)$

417 (18.9)

$908(41.1)$

$573(25.9)$

$3715(35.4)$

$6790(64.6)$

$1638(74.1)$

$897(40.6)$

6842 (65.1)

$3663(34.9)$
Table 1 (continued)

\begin{tabular}{|c|c|c|c|}
\hline Variables & $\begin{array}{l}2005 \\
(n=2211) \\
n(\%)\end{array}$ & $\begin{array}{l}2010 \\
(n=10,505) \\
n(\%)\end{array}$ & $\begin{array}{l}2014 \\
(n=10,849) \\
n(\%)\end{array}$ \\
\hline \multicolumn{4}{|l|}{ Health insurance } \\
\hline No & - & 9409 (89.6) & $9172(84.5)$ \\
\hline Yes & - & $1096(10.4)$ & $1677(15.5)$ \\
\hline \multicolumn{4}{|l|}{ Partner education } \\
\hline No formal education & $294(13.3)$ & $1265(12.0)$ & $1072(9.9)$ \\
\hline Primary & $1186(53.7)$ & $4845(46.1)$ & $4995(46.0)$ \\
\hline Secondary and higher & $731(33.0)$ & 4395 (41.9) & $4782(44.1)$ \\
\hline \multicolumn{4}{|l|}{ Contraceptive use } \\
\hline No & $1232(55.7)$ & $4716(44.9)$ & $4221(38.9)$ \\
\hline Traditional & $339(15.4)$ & $1790(17.0)$ & $2043(18.8)$ \\
\hline Modern & $640(28.9)$ & $3999(38.1)$ & $4585(42.3)$ \\
\hline \multicolumn{4}{|l|}{ Community-level factors } \\
\hline \multicolumn{4}{|l|}{ Community wealth } \\
\hline Low & $658(29.7)$ & $3706(35.3)$ & $3778(34.8)$ \\
\hline Middle & $841(38.1)$ & $3999(38.1)$ & $4426(40.8)$ \\
\hline High & $712(32.2)$ & $2800(26.6)$ & $2645(24.4)$ \\
\hline \multicolumn{4}{|l|}{ Community education } \\
\hline Low & $675(30.5)$ & $2810(26.8)$ & $3481(32.1)$ \\
\hline Middle & 749 (33.9) & $3668(34.9)$ & $3872(35.7)$ \\
\hline High & $787(35.6)$ & $4027(38.3)$ & $3496(32.2)$ \\
\hline \multicolumn{4}{|c|}{ Community decision-making } \\
\hline Low & $569(25.7)$ & $3643(34.7)$ & $3430(31.6)$ \\
\hline Middle & $723(32.7)$ & $3571(34.0)$ & $3576(33.0)$ \\
\hline High & 919 (41.6) & $3291(31.3)$ & $3843(35.4)$ \\
\hline \multicolumn{4}{|c|}{ Community workforce participation } \\
\hline Low & 788 (35.6) & $3388(32.3)$ & $3941(36.3)$ \\
\hline Middle & $751(34.0)$ & $3795(36.1)$ & $3897(35.9)$ \\
\hline High & $672(30.4)$ & $3322(31.6)$ & $3011(27.8)$ \\
\hline
\end{tabular}

HF Health facility

95\% CI: 1.13-1.43] and high [aOR: 1.44, 95\% CI: $1.29-$ 1.60] workforce participation levels.

At community level, in 2010, women from communities with high a high percentage of educated women were less likely [aOR: 0.83 , 95\% CI: 0.69-0.99] to use any contraceptive method compared with those from communities with a low percentage of educated women. Similarly, in 2014, women from communities with a middle [aOR: 0.84, 95\% CI: 0.72-0.97] and a high [aOR: 0.78, 95\% CI: 0.66-0.91] percentage of educated women were less likely to use any contraceptive methods. Additionally, it was observed in 2005 that women from communities with a middle percentage of women in workforce were more likely [aOR: $1.38,95 \% \mathrm{CI}: 1.06-1.81$ ] to use any contraceptive method compared with those from communities with a low percentage of women in workforce. 
Table 2 Multilevel logistic analysis of factors associated with contraceptive use in Cambodia

\begin{tabular}{|c|c|c|c|}
\hline \multirow[t]{2}{*}{ Variables } & \multirow{2}{*}{$\begin{array}{l}2005 \\
\text { Any method vs. no method } \\
\text { aOR }(95 \% \mathrm{Cl})\end{array}$} & \multirow{2}{*}{$\begin{array}{l}2010 \\
\text { Any method vs. no method } \\
\text { aOR }(95 \% \mathrm{Cl})\end{array}$} & \multirow{2}{*}{$\begin{array}{l}2014 \\
\text { Any method vs. no method } \\
\text { aOR }(95 \% \mathrm{Cl})\end{array}$} \\
\hline & & & \\
\hline \multicolumn{4}{|l|}{ Individual-level factors } \\
\hline \multicolumn{4}{|l|}{ Educational level } \\
\hline No formal education & 1.00 & 1.00 & 1.00 \\
\hline Primary & $1.12(0.85-1.47)$ & $1.14(1.01-1.29)$ & $1.17(1.02-1.34)$ \\
\hline Secondary+ & $1.34(0.91-1.96)$ & $1.43(1.22-1.68)$ & $1.23(1.05-1.44)$ \\
\hline \multicolumn{4}{|l|}{ Decision-making } \\
\hline Low & 1.00 & 1.00 & 1.00 \\
\hline Middle & $1.08(0.65-1.80)$ & $0.94(0.72-1.23)$ & $1.17(0.89-1.54)$ \\
\hline High & $1.09(0.66-1.77)$ & $0.99(0.77-1.25)$ & $1.21(0.94-1.54)$ \\
\hline \multicolumn{4}{|l|}{ Workforce participation } \\
\hline Low & 1.00 & 1.00 & 1.00 \\
\hline Medium & $1.27(0.99-1.62)$ & $1.14(1.01-1.28)$ & $1.27(1.13-1.43)$ \\
\hline High & $1.28(0.97-1.69)$ & $1.12(1.00-1.26)$ & $1.44(1.29-1.60)$ \\
\hline \multicolumn{4}{|l|}{ Age (years) } \\
\hline $15-24$ & 1.00 & 1.00 & 1.00 \\
\hline $25-34$ & $1.21(0.88-1.68)$ & $1.36(1.18-1.57)$ & $1.25(1.08-1.43)$ \\
\hline$\geq 35$ & $0.79(0.54-1.14)$ & $0.71(0.61-0.84)$ & $0.57(0.49-0.67)$ \\
\hline \multicolumn{4}{|l|}{ Religion } \\
\hline Buddhist & 1.00 & 1.00 & 1.00 \\
\hline Muslim & $0.88(0.41-1.91)$ & $0.93(0.64-1.37)$ & $0.89(0.63-1.26)$ \\
\hline Other & $0.74(0.39-1.41)$ & $0.78(0.57-1.08)$ & $1.15(0.87-1.53)$ \\
\hline \multicolumn{4}{|l|}{ Residence } \\
\hline Urban & 1.00 & 1.00 & 1.00 \\
\hline Rural & $0.89(0.67-1.18)$ & $0.97(0.80-1.16)$ & $1.25(1.05-1.49)$ \\
\hline \multicolumn{4}{|l|}{ Region } \\
\hline Plains & 1.00 & 1.00 & 1.00 \\
\hline Tonle Sap & $1.49(1.13-1.96)$ & $1.04(0.89-1.23)$ & $1.11(0.96-1.27)$ \\
\hline Plateau / Mountain & $1.69(1.23-2.32)$ & $0.99(0.83-1.18)$ & $1.05(0.90-1.23)$ \\
\hline Phnom Penh & $1.29(0.76-2.18)$ & $0.92(0.68-1.25)$ & $0.80(0.60-1.05)$ \\
\hline \multicolumn{4}{|l|}{ Age at first marriage } \\
\hline$\leq 16$ & 1.00 & 1.00 & 1.00 \\
\hline $17-20$ & $0.93(0.72-1.20)$ & $0.91(0.81-1.02)$ & $0.87(0.77-0.97)$ \\
\hline$\geq 21$ & $0.70(0.52-0.94)$ & $0.75(0.66-0.86)$ & $0.66(0.59-0.75)$ \\
\hline \multicolumn{4}{|l|}{ Total children ever born } \\
\hline 0 & 1.00 & 1.00 & 1.00 \\
\hline $1-2$ & $6.40(2.84-14.43)$ & 13.19 (8.70-19.99) & 13.58 (9.57-19.27) \\
\hline$\geq 3$ & $6.89(3.00-15.81)$ & 14.98(9.79-22.92) & $16.94(11.80-24.32)$ \\
\hline \multicolumn{4}{|l|}{ Wealth } \\
\hline Poor & 1.00 & 1.00 & 1.00 \\
\hline Middle & $1.23(0.93-1.62)$ & $1.09(0.96-1.24)$ & $0.96(0.84-1.09)$ \\
\hline Rich & $1.36(0.99-1.87)$ & $0.87(0.75-1.01)$ & $0.89(0.77-1.03)$ \\
\hline \multicolumn{4}{|l|}{ Media exposure } \\
\hline No & 1.00 & 1.00 & 1.00 \\
\hline Yes & $1.14(0.90-1.46)$ & $1.06(0.96-1.17)$ & $0.98(0.88-1.08)$ \\
\hline \multicolumn{4}{|l|}{ Perceived distance to HF } \\
\hline Big problem & 1.00 & 1.00 & 1.00 \\
\hline No problem & $0.98(0.79-1.21)$ & $1.03(0.93-1.13)$ & $1.03(0.92-1.11)$ \\
\hline
\end{tabular}


Table 2 (continued)

\begin{tabular}{|c|c|c|c|}
\hline \multirow[t]{2}{*}{ Variables } & \multirow{2}{*}{$\begin{array}{l}2005 \\
\text { Any method vs. no method } \\
\text { aOR }(95 \% \mathrm{Cl})\end{array}$} & \multirow{2}{*}{$\begin{array}{l}2010 \\
\text { Any method vs. no method } \\
\text { aOR }(95 \% \mathrm{Cl})\end{array}$} & \multirow{2}{*}{$\begin{array}{l}2014 \\
\text { Any method vs. no method } \\
\text { aOR }(95 \% \mathrm{Cl})\end{array}$} \\
\hline & & & \\
\hline \multicolumn{4}{|l|}{ Health insurance } \\
\hline No & - & 1.00 & 1.00 \\
\hline Yes & - & $0.90(0.79-1.03)$ & $1.25(1.10-1.41)$ \\
\hline \multicolumn{4}{|l|}{ Partner education } \\
\hline No formal education & 1.00 & 1.00 & 1.00 \\
\hline Primary & $1.22(0.90-1.66)$ & $1.16(1.00-1.34)$ & $0.99(0.85-1.37)$ \\
\hline Secondary and higher & $1.19(0.84-1.71)$ & $1.15(0.98-1.36)$ & $1.04(0.88-1.23)$ \\
\hline \multicolumn{4}{|l|}{ Community-level factors } \\
\hline \multicolumn{4}{|l|}{ Community wealth } \\
\hline Low & 1.00 & 1.00 & 1.00 \\
\hline Middle & $1.05(0.79-1.39)$ & $0.92(0.78-1.09)$ & $1.02(0.88-1.18)$ \\
\hline High & $0.92(0.62-1.38)$ & $0.87(0.68-1.10)$ & $1.10(0.87-1.39)$ \\
\hline \multicolumn{4}{|l|}{ Community education } \\
\hline Low & 1.00 & 1.00 & 1.00 \\
\hline Middle & $1.15(0.87-1.52)$ & $0.92(0.78-1.08)$ & $0.84(0.72-0.97)$ \\
\hline High & $0.97(0.72-1.34)$ & $0.83(0.69-0.99)$ & $0.78(0.66-0.91)$ \\
\hline \multicolumn{4}{|c|}{ Community decision-making } \\
\hline Low & 1.00 & 1.00 & 1.00 \\
\hline Middle & $1.03(0.78-1.36)$ & $1.05(0.90-1.23)$ & $1.01(0.88-1.17)$ \\
\hline High & $1.09(0.82-1.45)$ & $1.04(0.88-1.23)$ & $0.96(0.83-1.11)$ \\
\hline \multicolumn{4}{|c|}{ Community workforce participation } \\
\hline Low & 1.00 & 1.00 & 1.00 \\
\hline Middle & $1.38(1.06-1.81)$ & $1.08(0.92-1.27)$ & $0.95(0.82-1.09)$ \\
\hline High & $1.32(0.99-1.76)^{\ni}$ & $1.12(0.94-1.23)$ & $0.96(0.74-1.04)$ \\
\hline \multicolumn{4}{|l|}{ Measures of variation } \\
\hline Area variance $(95 \% \mathrm{Cl})$ & $0.23(0.10-0.57)$ & $0.28(0.23-0.36)$ & $0.18(0.13-0.24)$ \\
\hline ICC (\%) & 6.53 & 8.00 & 5.22 \\
\hline PCV (\%) & 23.33 & 3.45 & 14.29 \\
\hline MOR & 1.58 & 1.66 & 1.50 \\
\hline \multicolumn{4}{|l|}{ Model fit statistic } \\
\hline AIC & 2770.24 & $13,127.01$ & $13,487.78$ \\
\hline
\end{tabular}

7 borderline $p$-value, bold means $p$-value $<0.05$

aOR Adjusted odds ratio, CI Confidence internal, ICC Intraclass correlation coefficient, MOR Median odds ratio, PVC Proportional change in variance, AIC Akaike information criterion

\section{Empowerment factors associated with use of specific contraceptive methods}

Results from the multinomial logistic regression analyses are listed in Table 3. Results from model 4 are presented because the model had a better goodness of fit (i.e., lower AIC). Educational level was associated with increased odds of using both modern and traditional methods (with no method as base category) in 2010 and 2014. Having primary education was positively associated [aOR: 1.52, 95\% CI: 1.01-2.75] with using traditional contraceptive methods in 2005. In 2010, the odds of using traditional methods were higher among those with primary [aOR: 1.41, 95\% CI: 1.15-1.91], and secondary and tertiary education [aOR: 1.99, 95\% CI: 1.57-2.05] compared with those having no formal education. Additionally, the odds of using modern methods were high among those with primary [aOR: 1.21, 95\% CI: 1.06-1.38] and secondary and tertiary [aOR: 1.73, 95\% CI: 1.46-2.05] education. Similarly, having secondary and tertiary education was positively associated with use of traditional method [aOR: 1.46, 95\% CI: 1.15-1.85] compared with those having no formal education in 2014. Further, having primary [aOR: 1.23, 95\% CI: 1.06-1.42] and secondary and tertiary [aOR: 
Table 3 Multilevel multinomial logistic regression analysis of factors associated with contraceptive use in Cambodia based om 2005, 2010, and 2014 (Final Models only)

\begin{tabular}{|c|c|c|c|c|c|c|}
\hline \multirow[t]{2}{*}{ Variables } & \multicolumn{2}{|l|}{2005} & \multicolumn{2}{|l|}{2010} & \multicolumn{2}{|l|}{2014} \\
\hline & $\begin{array}{l}\text { Traditional vs } \\
\text { no method } \\
\text { RRR }(95 \% \mathrm{Cl})\end{array}$ & $\begin{array}{l}\text { Modern vs } \\
\text { no method } \\
\text { RRR }(95 \% \mathrm{Cl})\end{array}$ & $\begin{array}{l}\text { Traditional vs } \\
\text { no method } \\
\text { RRR }(95 \% \mathrm{Cl})\end{array}$ & $\begin{array}{l}\text { Modern vs } \\
\text { no method } \\
\text { RRR }(95 \% \mathrm{Cl})\end{array}$ & $\begin{array}{l}\text { Traditional vs } \\
\text { no method } \\
\text { RRR }(95 \% \mathrm{Cl})\end{array}$ & $\begin{array}{l}\text { Modern vs } \\
\text { no method } \\
\text { RRR }(95 \% \mathrm{Cl})\end{array}$ \\
\hline \multicolumn{7}{|c|}{ Individual-level factors } \\
\hline \multicolumn{7}{|c|}{ Educational level } \\
\hline $\begin{array}{l}\text { No formal educa- } \\
\text { tion }\end{array}$ & 1.00 & 1.00 & 1.00 & 1.00 & 1.00 & 1.00 \\
\hline Primary & $1.52(1.01-2.75)$ & $1.20(0.90-1.59)$ & $1.41(1.15-1.91)$ & $1.21(1.06-1.38)$ & $1.22(0.99-1.50)$ & $1.23(1.06-1.42)$ \\
\hline Secondary+ & $1.54(0.90-1.99)$ & $1.45(0.97-2.18)$ & $1.99(1.57-2.52)$ & $1.73(1.46-2.05)$ & $1.46(1.15-1.85)$ & $1.38(1.16-1.65)$ \\
\hline \multicolumn{7}{|l|}{ Decision-making } \\
\hline Low & 1.00 & 1.00 & 1.00 & 1.00 & 1.00 & 1.00 \\
\hline Middle & $1.32(0.58-1.17)$ & $1.11(0.66-1.88)$ & $1.36(0.91-2.04)$ & $1.01(0.76-1.34)$ & $1.22(0.85-1.75)$ & $1.26(0.94-1.70)$ \\
\hline High & $1.26(0.57-2.78)$ & $1.09(0.65-1.81)$ & $1.71(1.18-2.47)$ & $1.13(0.88-1.46)$ & $1.05(0.75-1.45)$ & $1.23(0.94-1.61)$ \\
\hline \multicolumn{7}{|c|}{ Workforce participation } \\
\hline Low & 1.00 & 1.00 & 1.00 & 1.00 & 1.00 & $1.19(0.99-1.44)$ \\
\hline Medium & $0.81(0.56-1.17)$ & $1.23(0.95-1.59)$ & $1.48(1.25-1.76)$ & $1.24(1.10-1.41)$ & $1.53(1.29-1.82)$ & $1.45(1.27-1.65)$ \\
\hline High & $1.33(0.91-1.94)$ & $1.38(1.02-1.87)$ & $1.49(1.27-1.74)$ & $1.25(1.10-1.41)$ & $1.69(1.45-1.96)$ & $1.72(1.52-1.94)$ \\
\hline \multicolumn{7}{|l|}{ Age (years) } \\
\hline $15-24$ & 1.00 & 1.00 & 1.00 & 1.00 & 1.00 & 1.00 \\
\hline $25-34$ & $1.08(0.67-1.75)$ & $1.24(0.88-1.75)$ & $1.52(1.23-1.87)$ & $1.53(1.32-1.79)$ & $1.31(1.08-1.59)$ & $1.38(1.19-1.61)$ \\
\hline$\geq 35$ & $0.83(0.48-1.41)$ & $0.75(0.51-1.11)$ & $0.93(0.73-1.17)$ & $0.70(0.59-0.83)$ & $0.60(0.48-0.75)$ & $0.48(0.40-0.57)$ \\
\hline \multicolumn{7}{|l|}{ Religion } \\
\hline Buddhist & 1.00 & 1.00 & 1.00 & 1.00 & 1.00 & 1.00 \\
\hline Muslim & $0.38(0.10-1.45)$ & $0.73(0.32-1.64)$ & $0.87(0.51-1.48)$ & $0.91(0.61-1.36)$ & $0.57(0.34-0.95)$ & $0.75(0.52-1.09)$ \\
\hline Other & $0.09(0.01-0.70)$ & $0.63(0.06-1.23)$ & $0.42(0.23-0.76)$ & $0.72(0.51-0.99)$ & $0.71(0.45-1.12)$ & $1.06(0.79-1.44)$ \\
\hline \multicolumn{7}{|l|}{ Residence } \\
\hline Urban & 1.00 & 1.00 & 1.00 & 1.00 & 1.00 & 1.00 \\
\hline Rural & $1.16(0.77-1.74)$ & $0.92(0.68-1.25)$ & $0.97(0.76-1.23)$ & $0.96(0.78-1.66)$ & $1.27(1.01-1.59)$ & $1.37(1.13-1.66)$ \\
\hline \multicolumn{7}{|l|}{ Region } \\
\hline Plains & 1.00 & 1.00 & 1.00 & 1.00 & 1.00 & 1.00 \\
\hline Tonle Sap & $1.67(1.13-2.47)$ & $1.68(1.25-2.24)$ & $0.81(0.66-0.99)$ & $0.98(0.83-1.16)$ & $1.14(0.95-1.37)$ & $1.15(0.99-1.34)$ \\
\hline $\begin{array}{l}\text { Plateau / Moun- } \\
\text { tain }\end{array}$ & $1.85(1.17-2.93)$ & $1.95(1.39-2.74)$ & $0.68(0.54-0.86)$ & $0.90(0.75-1.09)$ & $0.99(0.81-1.22)$ & $1.06(0.89-1.26)$ \\
\hline Phnom Penh & $5.46(2.71-10.99)$ & $2.33(1.27-4.29)$ & $1.18(0.82-1.70)$ & $0.99(0.72-1.38)$ & $1.79(1.29-2.49)$ & $1.06(0.78-1.45)$ \\
\hline \multicolumn{7}{|c|}{ Age at first marriage } \\
\hline$\leq 16$ & 1.00 & 1.00 & 1.00 & 1.00 & 1.00 & 1.00 \\
\hline $17-20$ & $1.13(0.77-1.67)$ & $0.96(0.73-1.25)$ & $1.15(0.96-1.38)$ & $0.94(0.83-1.06)$ & $1.21(1.02-1.43)$ & $0.92(0.81-1.04)$ \\
\hline$\geq 21$ & $1.41(0.92-2.17)$ & $0.76(0.55-1.04)$ & $1.04(0.86-1.27)$ & $0.76(0.51-0.87)$ & $1.04(0.86-1.24)$ & $0.67(0.58-0.77)$ \\
\hline \multicolumn{7}{|c|}{ Total children ever born } \\
\hline 0 & 1.00 & 1.00 & 1.00 & 1.00 & 1.00 & 1.00 \\
\hline $1-2$ & $6.39(2.31-17.72)$ & $8.90(3.88-20.43)$ & $5.57(3.84-8.07)$ & $\begin{array}{l}18.21(11.97- \\
27.70)\end{array}$ & $5.67(4.21-7.64)$ & $\begin{array}{l}20.28(14.23- \\
28.91)\end{array}$ \\
\hline$\geq 3$ & $8.92(3.11-25.56)$ & $10.31(4.39-24.17)$ & $6.52(4.41-9.65)$ & $\begin{array}{l}21.53(13.99- \\
33.12)\end{array}$ & $8.25(5.95-11.41)$ & $\begin{array}{l}28.92(19.97- \\
41.88)\end{array}$ \\
\hline \multicolumn{7}{|l|}{ Wealth } \\
\hline Poor & 1.00 & 1.00 & 1.00 & 1.00 & 1.00 & 1.00 \\
\hline Middle & $1.20(0.80-1.80)$ & $1.26(0.94-1.69)$ & $1.33(1.09-1.63)$ & $1.15(1.00-1.32)$ & $0.97(0.80-1.18)$ & $0.94(0.82-1.09)$ \\
\hline Rich & $1.27(0.80-2.01)$ & $1.42(1.01-1.98)$ & $1.20(0.97-1.49)$ & $0.89(0.76-1.04)$ & $1.05(0.85-1.29)$ & $0.91(0.77-1.06)$ \\
\hline \multicolumn{7}{|l|}{ Media exposure } \\
\hline No & 1.00 & 1.00 & 1.00 & 1.00 & 1.00 & 1.00 \\
\hline
\end{tabular}


Table 3 (continued)

\begin{tabular}{|c|c|c|c|c|c|c|}
\hline \multirow[t]{2}{*}{ Variables } & \multicolumn{2}{|l|}{2005} & \multicolumn{2}{|l|}{2010} & \multicolumn{2}{|l|}{2014} \\
\hline & $\begin{array}{l}\text { Traditional vs } \\
\text { no method } \\
\text { RRR }(95 \% \mathrm{Cl})\end{array}$ & $\begin{array}{l}\text { Modern vs } \\
\text { no method } \\
\text { RRR }(95 \% \mathrm{Cl})\end{array}$ & $\begin{array}{l}\text { Traditional vs } \\
\text { no method } \\
\text { RRR }(95 \% \mathrm{Cl})\end{array}$ & $\begin{array}{l}\text { Modern vs } \\
\text { no method } \\
\text { RRR }(95 \% \mathrm{Cl})\end{array}$ & $\begin{array}{l}\text { Traditional vs } \\
\text { no method } \\
\text { RRR }(95 \% \mathrm{CI})\end{array}$ & $\begin{array}{l}\text { Modern vs } \\
\text { no method } \\
\text { RRR }(95 \% \mathrm{Cl})\end{array}$ \\
\hline Yes & $1.32(0.93-1.89)$ & $1.22(0.94-1.57)$ & $1.02(0.88-1.19)$ & $1.06(0.95-1.18)$ & $1.00(0.87-1.16)$ & $0.98(0.88-1.09)$ \\
\hline \multicolumn{7}{|c|}{ Perceived distance to HF } \\
\hline Big problem & 1.00 & 1.00 & 1.00 & 1.00 & 1.00 & 1.00 \\
\hline No problem & $1.65(1.21-2.25)$ & $1.09(0.87-1.36)$ & $1.30(1.12-1.50)$ & $1.09(0.98-1.21)$ & $1.24(1.08-1.42)$ & $1.08(0.97-1.20)$ \\
\hline \multicolumn{7}{|l|}{ Health insurance } \\
\hline No & - & - & 1.00 & 1.00 & 1.00 & 1.00 \\
\hline Yes & - & - & $0.76(0.61-0.95)$ & $0.85(0.74-0.99)$ & $0.95(0.79-1.14)$ & $1.23(1.07-1.41)$ \\
\hline \multicolumn{7}{|l|}{ Partner education } \\
\hline $\begin{array}{l}\text { No formal educa- } \\
\text { tion }\end{array}$ & 1.00 & 1.00 & 1.00 & 1.00 & 1.00 & 1.00 \\
\hline Primary & $0.88(0.56-1.37)$ & $1.19(0.86-1.63)$ & $1.56(1.21-2.02)$ & $1.23(1.06-1.43)$ & $1.26(0.98-1.62)$ & $1.04(0.88-1.22)$ \\
\hline $\begin{array}{l}\text { Secondary and } \\
\text { higher }\end{array}$ & $1.30(0.79-2.14)$ & $1.28(0.88-1.87)$ & $2.11(1.61-2.76)$ & $1.34(1.13-1.58)$ & $1.51(1.16-1.97)$ & $1.17(0.98-1.40)$ \\
\hline \multicolumn{7}{|c|}{ Community-level factors } \\
\hline \multicolumn{7}{|c|}{ Community wealth } \\
\hline Low & 1.00 & 1.00 & 1.00 & 1.00 & 1.00 & 1.00 \\
\hline Middle & $0.83(0.55-1.26)$ & $0.99(0.74-1.35)$ & $1.51(1.20-1.91)$ & $1.00(0.84-1.19)$ & $1.11(0.90-1.37)$ & $1.04(0.89-1.23)$ \\
\hline High & $0.99(0.56-2.65)$ & $0.92(0.60-1.41)$ & $1.86(1.35-2.58)$ & $1.01(0.78-1.30)$ & $1.35(0.99-1.85)$ & $1.22(0.94-1.65)$ \\
\hline \multicolumn{7}{|c|}{ Community education } \\
\hline Low & 1.00 & 1.00 & 1.00 & 1.00 & 1.00 & 1.00 \\
\hline Middle & $1.41(0.94-2.11)$ & $1.25(0.93-1.68)$ & $1.16(0.92-1.46)$ & $0.94(0.79-1.12)$ & $1.37(1.12-1.68)$ & $0.91(0.78-1.06)$ \\
\hline High & $1.05(0.67-1.65)$ & $0.98(0.71-1.37)$ & $1.12(0.87-1.44)$ & $0.84(0.70-1.02)$ & $1.42(1.14-1.76)$ & $0.86(0.72-1.02)$ \\
\hline \multicolumn{7}{|c|}{ Community decision-making } \\
\hline Low & 1.00 & 1.00 & 1.00 & 1.00 & 1.00 & 1.00 \\
\hline Middle & $0.91(0.61-1.37)$ & $0.99(0.74-1.35)$ & $0.76(0.62-0.94)$ & $0.98(0.83-1.16)$ & $1.01(0.84-1.22)$ & $1.01(0.87-1.19)$ \\
\hline High & $1.20(0.80-1.80)$ & $1.13(0.83-1.53)$ & $0.65(0.52-0.82)$ & $0.93(0.78-1.11)$ & $1.10(0.91-1.34)$ & $0.99(0.84-1.16)$ \\
\hline \multicolumn{7}{|c|}{ Community workforce participation } \\
\hline Low & 1.00 & 1.00 & 1.00 & 1.00 & 1.00 & 1.00 \\
\hline Middle & $0.74(0.50-1.10)$ & $1.29(0.97-1.73)$ & $0.92(0.74-1.14)$ & $1.06(0.90-1.26)$ & $1.31(1.07-1.60)$ & $1.02(0.87-1.19)$ \\
\hline High & $0.88(0.58-1.32)$ & $1.29(0.95-1.76)$ & $0.82(0.65-1.04)$ & $1.07(0.89-1.29)$ & $1.41(1.12-1.77)$ & $0.97(0.81-1.18)$ \\
\hline \multicolumn{7}{|c|}{ Measures of variation } \\
\hline $\begin{array}{l}\text { Area variance } \\
(95 \% \mathrm{Cl})\end{array}$ & $0.55(0.26-1.59)$ & $0.32(0.15-0.67)$ & $0.37(0.27-0.51)$ & $0.30(0.24-0.39)$ & $0.24(0.17-0.36)$ & $0.20(0.15-0.28)$ \\
\hline ICC (\%) & 14.32 & 8.86 & 10.11 & 10.31 & 6.80 & 5.73 \\
\hline PCV (\%) & 51.33 & 33.33 & 51.95 & 10.71 & 51.02 & 5.26 \\
\hline MOR & 2.03 & 1.72 & 1.79 & 1.70 & 1.60 & 1.53 \\
\hline \multicolumn{7}{|l|}{ Model fit statistic } \\
\hline $\mathrm{AIC}$ & 4258.33 & 4258.33 & $19,634.60$ & $19,634.60$ & $20,406.73$ & $20,406.73$ \\
\hline
\end{tabular}

RRR Relative risk ratio, CI Confidence internal, ICC Intraclass correlation coefficient, MOR Median odds ratio, PVC Proportional change in variance, AIC Akaike information criterion

1.38, 95\% CI: 1.16-1.65] education was associated with increased likelihood of using modern contraceptive methods.

Having a high decision making power was associated with use of traditional contraceptive methods in 2010
[aOR: 1.71, 95\% CI: 1.18-2.47] compared with those having low decision making power.

Across all survey waves, having high work participation was associated with increased odds of using modern contraceptive methods (Table 3). However, having medium workforce participation level was associated with use 
of both traditional methods and modern methods only in 2010 [aOR: 1.48, 95\% CI: $1.25-1.76$ and aOR: 1.24, 95\% CI: $1.10-1.31$, for traditional and modern methods, respectively] and 2014 [aOR: 1.53, 95\% CI: 1.29-1.82 and aOR: 1.45 , 95\% CI: $1.27-1.65$, for traditional and modern methods, respectively] surveys.

At the community level, women from communities with a middle [aOR: 1.37, 95\% CI: 1.12-1.68] and high [aOR: 1.42, 95\% CI: 1.14-1.76] percentage of educated women were more likely to use traditional methods compared to those from communities with a low percentage of educated women in 2014 survey. Meanwhile, in women from communities with a middle [aOR: 0.76, 95\% CI: 0.62-0.94] and high [aOR: 0.65, 95\% CI: 0.52-0.82] percentage of women having decision making powers were less likely to use traditional methods in 2010 survey.

\section{Other factors associated with of contraceptive methods}

Tables 2 and 3 further reveals other factors that were associated with use of any contraceptive methods, and specific contraceptive method, respectively.

Among others, across all survey waves, older age at first marriage was associated with reduced likelihood of using any contraceptive method while having $>1$ child was associated with increased odds of using any contraceptive methods. In 2014, those that lived in rural areas were more likely to use any contraceptive methods. Regional variations were observed in 2005 in terms of use of any contraceptive methods while this variation was not significant in the other survey waves. Having a health insurance was associated with increased odds of using any contraceptive methods in 2014. Having a partner with primary education was associated with increased odds of using any contraceptive methods in 2010 (Table 2).

Women aged $\geq 35$ years were less likely to use modern methods in 2010 while those aged 25-34 were more likely to use both traditional and modern methods compared with women aged $<25$ years. On the other hand, in 2014 , women aged $\geq 35$ years were less likely to use both traditional and modern contraceptive methods compared with those aged $<25$ years. Other factors that were associated with specific type of contraceptive method use included; religion, area of residence, region, age at first marriage, number of children ever born, distance to health facility, and having a health insurance.

\section{Random effects}

Measures of variation for the binary and 3-level outcomes are listed in Tables 2 and 3, respectively. The final models revealed significant variances for the outcomes in all survey waves. The MOR for all the survey waves for the binary outcome displayed the effects of community heterogeneity indicating that if a woman moved to a community with a high probability of using any contraceptives, the median increase in the odds of using any contraceptives would be 1.58 in 2004, while 1.66 in 2010, and 1.50 in 2014 (Table 2). Residual heterogeneity in the outcomes was observed as seen by significant variances in all the final models as well as the ICCs that displayed that there were still some unmeasured community factors, that could influence contraceptive use, that were not included in the current analysis.

\section{Discussion}

This study examined the influence of women empowerment factors at both individual- and community- level on contraceptive use among married Cambodian women. Further, the study examined other relevant individualand community- level factors that may be associated with contraceptive use among Cambodian women. Notably, educational attainment and participating in workforce were associated with increased likelihood of using any contraceptive method as well as specific contraceptive methods particularly in 2010 and 2014. The study also demonstrated that there are other unmeasured community factors that may influence contraceptive use among Cambodian married women.

It was observed that the use of modern methods increased over the survey waves from 2005 at 29.0 to $42.3 \%$ in 2014. FP programs in Cambodia have focused on improving awareness and knowledge, building capacity of midwives to provide contraceptive choices, increasing FP choices through community-based distribution of contraceptives, and enhancing a secure supply of commodities $[39,40]$. Therefore, the increase in the use of modern methods may underline that the efforts and programs aimed at improving access to reproductive health care in Cambodia are making significant strides. However, more needs to be done as the reported $42.3 \%$ (2014) is relatively lower than the regional rate (68.0\%) reported for Asia in 2015 [41]. As such, it may be prudent for Cambodia to learn lessons from other Asian countries that are making good progress with regards to contraceptive use.

Empowering women has been shown to influence their health behaviors in previous studies [42, 43]. In the current analysis, it was revealed that educated women and those involved in workforce were more likely to use contraceptive methods. Educated women are better informed about the various methods available for fertility control and may have greater geographical and financial access to contraception and overall reproductive health services [44]. Participating in workforce may empower women economically. Working women are more likely to have access to their own spending money hence they 
have a greater opportunity to use those funds towards family planning and reproductive health service utilization [45]. The results of these empowerment measures were similar even after examining their associations with specific types of contraceptive methods. Findings from the current research revealed the importance of women's education attainment and workforce involvement in Cambodia with respect to the use of contraceptives. No significant findings were observed for decision making and contraceptive use albeit in 2010 where it was associated with the use of traditional contraceptive methods. Previous research in Cambodia identified women's access to new knowledge and abilities, which technically helped them to be involved in income-generating activities, as a key empowerment aspect mentioned by Cambodian women [46]. This may partly explain why educational attainment and workforce involvement had greater influences on the use of contraceptives than decision making.

Our findings suggested that women from communities with a high percentage of women in workforce were more likely to use any contraceptive method. It was also observed that women from communities with a high percentage of educated women were less likely to use any contraceptive methods. The significant effect of community education disappeared when the multinomial models were run to examine the association with specific type of contraceptive method and community-level women's decision making power then became negatively associated with contraceptive use. The results about the negative relationship between some community SES factors and contraceptive use is consistent to a Zimbabwean study [47], and a multi-country study that observed a negative association between educational attainment in community and contraceptive use [48]. They suggested that the level of education does not mediate the pronatalist norms prevalent among women in local communities. Although unexpected, this finding may be partially explained by the fact that social and cultural norms may still play an important role in influencing contraceptive use within communities [49]. As such, women in communities where negative influence on contraceptive use exist may be discouraged to adopt the contraceptives. More research is needed to understand such relationships.

Several other individual-level factors were considered. Older women ( $>34$ years) were less likely to use contraceptives while those aged between 25 and 34 were more likely to use contraceptives. The findings are in line with results reported in Iran [50]. Older women's awareness regarding their declining fertility could be one of the reasons why they are less likely to use both contraceptive methods. Consistent to previous research [51], age at first marriage was associated with contraceptive use with those aged $\geq 21$ years at first marriage being less likely to use contraceptives. Women who married later in life may have the desire to bear children in the earliest time possible therefore, they may not prefer to use any contraceptive methods. Regional variations were observed in terms of contraceptive use. Additionally, in contrast to previous research $[50,51]$, women from rural areas were more likely to use contraceptives. In Cambodia, programs relating to reproductive health may be specifically promoted in the rural areas than urban areas and this may explain the findings in the current study. It was observed that in 2010, having health insurance was negatively associated with contraceptive use while in 2014 there was a positive association. Continuous improvements to health insurance system over time could be the reason for the observed differences between 2010 and 2014. Women that did not perceive distance to the nearest health facility as a problem were more likely to use contraceptive methods. This underscores the importance of improving health care services access as it is a precursor to accessing reliable information including those related to contraceptive use.

\section{Policy/program implications}

First, strategies aiming at improving reproductive health in Cambodia should aim to integrate with efforts/programs that are geared to empower women as this may ultimately help improve utilization of contraceptives. Second, the study further revealed that other unmeasured community factors may influence contraceptive use among Cambodian women suggesting the need for public health programs to profile communities when designing or formulating their FP policies/programs. This may help in the development of tailored programs that may eventually be effective. Third, regional variations were observed in terms of contraceptive use suggesting that programs should focus on regions that are lagging behind. Fourth, improving contraceptive use in Cambodia requires a multifaceted approach with both the individual- and community- level factors identified in the current analysis being crucial to the implementation of effective programs.

\section{Strengths and limitations}

The study used three survey waves with nationally representative samples which allowed for generalizability of the results to the wider population of Cambodian married women. Additionally, the findings on the association between empowerment variables and contraceptive use across the survey waves help to strengthen the relationships observed. The assessment of variables at different levels allowed the study to account for community 
differences as well as identify the existence of other unmeasured community factors which is important in the design of public health programs and future research, respectively. However, the study design precludes inferences of causality. There were other factors, as observed, that may account for variation in contraceptive use (such a community outreach, engagement, and mobilization efforts) that were not included in the survey.

\section{Conclusion}

The factors influencing use of contraceptive methods (traditional or modern) among married Cambodian women operate at both individual and community level. Efforts to promote modern contraceptive use should aim at empowering women with a particular focus on improving access to education and employment opportunities. We recommend that through the avenues of education and increased awareness on personal rights, women would have a greater ability to negotiate with their husbands/partners to come to conclusions on contraceptive use that is best for both sides. Further, increasing labor market opportunities to give women economic independence. This could empower women and change social constructs for a more gender-equal world could have far-reaching positive effects that stem beyond improvements on reproductive health behaviors. To highlight the underlying cultural themes at work, as well as other unmeasured community factors, more research should be incorporated into future research for a more complete picture.

\section{Abbreviations \\ FP: Family planning; CDHS: Cambodia demographic and health survey; EA: Enumeration area; aOR: Adjusted odds ratio; Cl: Confidence interval; ICC: Intraclass correlation coefficient; PCV: Proportional change in variance; MOR: Median odds ratio; AIC: Akaike information criterion; DHS: Demographic and health survey.}

\section{Acknowledgements}

We acknowledge the International Classification of Functioning Disability and Health (ICF) for the permission to use the MDHS data set for analysis.

\section{Authors' contributions}

$Y$-CC and DL designed the study. ON analyzed the data and wrote the manuscript. Y-CC, K-YC, and DL revised the draft. The authors read and approved the final manuscript.

\section{Funding}

Ministry of Science and Technology, Taiwan: Grant No. MOST 106-2410-H-038017- to Dr. Ying-Chih Chuang.

\section{Availability of data and materials}

The study used, with permission, data from the International Classification of Functioning, Disability, and Health (ICF). The data is publicly available upon request from the ICF on (https://dhsprogram.com/data/available-datasets. $\mathrm{cfm})$.

\section{Ethics approval and consent to participate}

The survey protocol was reviewed and approved by the Cambodia National Ethical Committee for Health Research. Before each interview was conducted, a verbal informed consent was sought by each interviewer reading a prescribed statement to the respondent and recording in the questionnaire whether or not the respondent consented (or provided assent on behalf of minors). Then the interviewer signed his or her name attesting to the fact that he/she read the consent statement to the respondent. The method of collecting consent has been standardized in the DHS survey to ensure consistency as some participants are not able to write. Clearance to analyze the data was provided by the Demographic Health Survey (DHS) program. The data is publicly available and may be requested from the DHS program through https ://dhsprogram.com/data/available-datasets.cfm.

\section{Consent for publication}

Not applicable.

\section{Competing interests}

The author declare that they have no competing interests.

\section{Author details}

${ }^{1}$ School of Public Health, Taipei Medical University, Taipei City, Taiwan 110.

${ }^{2}$ Institute of Health and Wellbeing, University of Glasgow, Glasgow, UK.

Received: 19 March 2020 Accepted: 29 November 2020

Published online: 06 January 2021

\section{References}

1. Cleland J, Conde-Agudelo A, Peterson H, Ross J, Tsui A. 2 contraception and health. Lancet. 2012;380:149-56.

2. Black RE, Levin C, Walker N, Chou D, Liu L, Temmerman M, et al. Reproductive, maternal, newborn, and child health: key messages from disease control priorities 3rd edition. Lancet. 2016;388(10061):2811-24.

3. Elmusharaf K, Byrne E, O'Donovan D. Strategies to increase demand for maternal health services in resource-limited settings: challenges to be addressed. BMC Public Health. 2015;15(1):1-0.

4. World Health Organization. Neonatal and perinatal mortality: country, regional and global estimates: World Health Organization; 2006. https:// apps.who.int/iris/handle/10665/43444. Accessed 17 Oct 2020

5. DaVanzo J, Hale L, Razzaque A, Rahman M. Effects of interpregnancy interval and outcome of the preceding pregnancy on pregnancy outcomes in Matlab, Bangladesh. BJOG: Int J Gynecol Obstet. 2007;114(9):1079-87.

6. A Ahmed S, Li Q, Liu L, Tsui AO. Maternal deaths averted by contraceptive use: an analysis of 172 countries. Lancet. 2012;380(9837):111-25.

7. Chola L, McGee S, Tugendhaft A, Buchmann E, Hofman K. Scaling up family planning to reduce maternal and child mortality: the potential costs and benefits of modern contraceptive use in South Africa. PLoS One. 2015;10(6):e0130077.

8. Organization WH. Mother-baby package: implementing safe motherhood in countries: practical guide: World Health Organization; 1996. https ://www.who.int/maternal_child_adolescent/documents/who_dhe_ msm 9411/en/. Accessed 17 Oct 2020

9. Atmadja S, Gumilar G. Update health policy decision making in safe motherhood regional issue. ADI J Recent Innov (AJRI). 2020;1 (2 Maret):93-7.

10. Santora E. The impact of the safe motherhood initiative from 1987 to 2000. Embryo project encyclopedia; 2020.

11. Abate MG, Tareke AA. Individual and community level associates of contraceptive use in Ethiopia: a multilevel mixed effects analysis. Arch Public Health. 2019;77:46.

12. Starbird E, Norton M, Marcus R. Investing in family planning: key to achieving the sustainable development goals. Glob Health Sci Pract. 2016;4(2):191-210.

13. Ahmed S, Choi Y, Rimon JG, Alzouma S, Gichangi P, Guiella G, et al. Trends in contraceptive prevalence rates in sub-Saharan Africa since the 2012 London summit on family planning: results from repeated cross-sectional surveys. Lancet Glob Health. 2019;7(7):e904-11. 
14. Osotimehin B. Family planning as a critical component of sustainable global development. Glob Health Action. 2015;8:29978.

15. Behrman JR, Kohler HP, Watkins SC. Social networks and changes in contraceptive use over time: evidence from a longitudinal study in rural Kenya. Demography. 2002;39(4):713-38.

16. Bongaarts J, Watkins SC. Social interactions and contemporary fertility transitions. Popul Dev Rev. 1996;22(4):639-82.

17. Maharaj P, Cleland J. Women on top: the relative influence of wives and husbands on contraceptive use in KwaZulu-Natal. Women Health. 2005;41(2):31-41.

18. Hameed W, Azmat SK, Ali M, Sheikh Ml, Abbas G, Temmerman M, et al. Women's empowerment and contraceptive use: the role of independent versus Couples' decision-making, from a lower middle income country perspective. PLoS One. 2014;9(8):e104633.

19. Upadhyay UD, Gipson JD, Withers M, Lewis S, Ciaraldi EJ, Fraser A, et al. Women's empowerment and fertility: a review of the literature. Soc Sci Med. 2014;115:111-20.

20. Duflo E. Women empowerment and economic development. J Econ Lit. 2012;50(4):1051-79.

21. Gram L, Morrison J, Sharma N, Shrestha B, Manandhar D, Costello A, et al. Validating an agency-based tool for measuring women's empowerment in a complex public health trial in rural Nepal. J Hum Dev Capab. 2017;18(1):107-35.

22. Rahman MM, Mostofa MG, Hoque MA. Women's household decisionmaking autonomy and contraceptive behavior among Bangladeshi women. Sex Reprod Healthc. 2014;5(1):9-15

23. Willis B, Onda S, Stoklosa HM. Causes of maternal and child mortality among Cambodian sex workers and their children: a cross sectional study. BMC Public Health. 2016;16(1):1176.

24. Duff P, Evans JL, Stein ES, Page K, Maher L. Young Women's health study C. high pregnancy incidence and low contraceptive use among a prospective cohort of female entertainment and sex workers in Phnom Penh, Cambodia. BMC Pregnancy Childbirth. 2018;18(1):128.

25. Samandari G, Speizer IS, O'Connell K. The role of social support and parity on contraceptive use in Cambodia. Int Perspect Sex Reprod Health. 2010;36(3):122-31.

26. Lai S-L, Tey N-P. Contraceptive use in Cambodia: does household decision-making power matter? Cult Health Sex. 2020;22(7):778-93.

27. Ahinkorah BO, Seidu A-A, Appiah F, Budu E, Adu C, Aderoju YBG, et al. Individual and community-level factors associated with modern contraceptive use among adolescent girls and young women in Mali: a mixed effects multilevel analysis of the 2018 Mali demographic and health survey. Contraception Reprod Med. 2020;5(1):27.

28. Nieuwendyk LM, Belon AP, Vallianatos H, Raine KD, Schopflocher D, Spence JC, et al. How perceptions of community environment influence health behaviours: using the analysis grid for environments linked to obesity framework as a mechanism for exploration. Health Promot Chronic Dis Prev Can. 2016;36(9):175-84

29. Short SE, Mollborn S. Social determinants and health behaviors: conceptual frames and empirical advances. Curr Opin Psychol. 2015;5:78-84.

30. Directorate General for Health/Cambodia, and ICF International. Cambodia demographic and health survey 2014. Phnom Penh: National Institute of Statistics/Cambodia, Directorate General for Health/Cambodia, and ICF International; 2015.

31. National Institute of Statistics, Directorate General for Health, and ICF Macro. Cambodia demographic and health survey 2010. Phnom Penh: National Institute of Statistics, Directorate General for Health, and ICF Macro; 2011

32. National Institute of Public Health/Cambodia, National Institute of Statistics/Cambodia, and ORC Macro. Cambodia demographic and health survey 2005. Phnom Penh: National Institute of Public Health/Cambodia, National Institute of Statistics/Cambodia, and ORC Macro; 2006.

33. National Institute of Statistics/Cambodia, Directorate General for Health/ Cambodia, ICF International. Cambodia demographic and health survey 2014. Phnom Penh: National Institute of Statistics/Cambodia, Directorate General for Health/Cambodia, and ICF International; 2015.

34. National Institute of Statistics/Cambodia, Directorate General for Health/ Cambodia, ICF International. Cambodia demographic and health survey
2010. Phnom Penh: National Institute of Statistics/Cambodia, Directorate General for Health/Cambodia, and ICF International; 2010.

35. National Institute of Statistics/Cambodia, Directorate General for Health/ Cambodia, ICF International. Cambodia demographic and health survey 2005. Phnom Penh: National Institute of statistics/Cambodia, directorate general for health/Cambodia, And ICF international; 2005.

36. Ram F, Shekhar C, Chowdhury B. Use of traditional contraceptive methods in India \& its socio-demographic determinants. Indian J Med Res. 2014;140 Suppl(Suppl 1):S17-28.

37. Yaya S, Uthman OA, Ekholuenetale M, Bishwajit G. Women empowerment as an enabling factor of contraceptive use in sub-Saharan Africa: a multilevel analysis of cross-sectional surveys of 32 countries. Reprod Health. 2018;15(1):214.

38. ICF International. Demographic and health survey sampling and household listing manual. MEASURE DHS. Calverton: ICF International; 2012.

39. UNFPA. Cambodia Country Programme Brief on Family Planning. https:// cambodia.unfpa.org/en/publications/cambodia-country-programmebrief-family-planning. Accessed 17 Oct 2020

40. Cambodia: identifying actions for scaling up long-acting reversible contraceptives. World Health Organization Regional Office for the Western Pacific; 2017. Licence: CC BY-NC-SA 3.0 IGO. https://apps.who.int/iris/bitst ream/handle/10665/259992/9789290618218_eng.pdf?sequence=1\&isAll owed $=\mathrm{y}$. Accessed 17 Oct 2020.

41. United Nations, Department of Economic and Social Affairs, Population Division (2015). Trends in Contraceptive Use Worldwide 2015 (ST/ESA/ SER.A/349). https://www.un.org/en/development/desa/population/publi cations/pdf/family/trendsContraceptiveUse2015Report.pdf. Accessed 17 Oct 2020.

42. Tiruneh FN, Chuang K-Y, Chuang Y-C. Women's autonomy and maternal healthcare service utilization in Ethiopia. BMC Health Serv Res. 2017;17(1):718

43. Moonzwe Davis L, Schensul SL, Schensul JJ, Verma RK, Nastasi BK, Singh R. Women's empowerment and its differential impact on health in low-income communities in Mumbai, India. Glob Public Health. 2014:9(5):481-94.

44. Musick K, England P, Edgington S, Kangas N. Education differences in intended and unintended fertility. Soc Forces. 2009;88(2):543-72.

45. Reed E, Donta B, Dasgupta A, Ghule M, Battala M, Nair S, et al. Access to money and relation to women's use of family planning methods among young married women in rural India. Matern Child Health J. 2016;20(6):1203-10.

46. Doneys P, Doane DL, Norm S. Seeing empowerment as relational: lessons from women participating in development projects in Cambodia. Dev Pract. 2019;30(2):1-13.

47. Ngome E, Odimegwu C. The social context of adolescent women's use of modern contraceptives in Zimbabwe: a multilevel analysis. Reprod Health. 2014;11(1):64.

48. Mutumba M, Wekesa E, Stephenson R. Community influences on modern contraceptive use among young women in low and middle-income countries: a cross-sectional multi-country analysis. BMC Public Health. 2018;18(1):430.

49. Walston NJW, DC: Policy Project. Country analysis of family planning and HIV/AIDS programs: Cambodia 2005;26:2006. http://www.policyproject. com/pubs/countryreports/CamFP-HIV_analysis.pdf. Accessed 17 Oct 2020.

50. Nouhjah S, Amiri E, Khodai A, Yazdanpanah A, Nadi Baghu M. Popular contraceptive methods in women aged 35 years and older attending health centers of 4 cities in Khuzestan province, Iran. Iran Red Crescent Med J. 2013;15(10):e4414-e.

51. Haq I, Sakib S, Talukder A. Sociodemographic factors on contraceptive use among ever-married women of reproductive age: evidence from three demographic and health surveys in Bangladesh. Med Sci (Basel). 2017;5(4):31.

\section{Publisher's Note}

Springer Nature remains neutral with regard to jurisdictional claims in published maps and institutional affiliations. 